\title{
A Case Report of Sleep-Related Painful Erection Successfully Treated with Paroxetine
}

\author{
Siwei Lin, Haiwang Lu, Desheng Wang, Jie Wang, Bo Dai, and Bin Bin* \\ Department of Andrology, First Affiliated Hospital of Guxangxi University of Traditional Chinese Medicine, Nanning, 530023, China
}

*Corresponding authors: Bin Bin, Department of Andrology, First Affiliated Hospital of Guxangxi University of Traditional Chinese Medicine, Nanning, 530023, China, E-mail: billbinn@sina.com

Received: 26 Sep, 2021 | Accepted: 21 Oct, 2021 | Published: 29 Oct, 2021

Citation: Lin S, Lu H, Wang D, Wang J, Dai B, et al. (2021) A Case Report of Sleep-Related Painful Erection Successfully Treated with Paroxetine. J Clin Case Stu 6(5): dx.doi.org/10.16966/2471-4925.238

Copyright: (C) 2021 Lin S, et al. This is an open-access article distributed under the terms of the Creative Commons Attribution License, which permits unrestricted use, distribution, and reproduction in any medium, provided the original author and source are credited.

\section{Abstract}

Sleep-Related Painful Erection (SRPE) is a rare condition characterized by recurrent, painful penile erections occurring when awakening from the Rapid Eye Movement (REM) sleep stage. The cause of SRPE is still unknown, the therapeutic strategies still in an expert-based opinion phase and there is no consensus yet. We present a case of a 23-year-old patient suffering from SRPE for 1 year, the smart bracelet which has a sleep monitoring function showed his sleep was fragmented by awakenings at the end of all the REM period. Several treatments such as tamsulosin and highfrequency hyperthermia therapy and Chinese herbal medicine did not prompt any improvement of his condition, but after taking a single daily dose of paroxetine $20 \mathrm{mg}$ for twelves weeks, both the frequency and intensity of SRPE gradually decreased. Even though the antidepressants to which paroxetine belongs were included as one of the abandoned treatments in recent review, in our case, paroxetine showed a long-term and stable effect on patients with SRPE, it indicates that the therapeutic effect of paroxetine on SRPE deserves further study and observation.

Abbreviations: SRPE: Sleep Related Painful Erection; REM: Rapid Eye Movement; PSG: Polysomnography; NPT-R: Nocturnal Penile Tumescence And Rigidity; PED5: Phosphodiesterase Type 5; cGMP: Cyclic Guanosine Monophosphate; NPT: Nocturnal Penile Tumescence

Keywords: Sleep related painful erection; SRPE; Paroxetine; Antidepressants

\section{Introduction}

Sleep-related painful erection (SRPE) is a rare disorder defined as "penile pain that occurs during erections, typically during rapid eye movement episodes" [1], patients with SRPE often woke up several times a night with a deep painful erection, which results in serious sleep deprivation, leads to anxiety, depression, irritable and fatigue. Generally, patients have normal erections during intercourse and wakefulness. To date, only 47 cases have been reported worldwide [2], the exact etiology remains unknown, various treatments have been tried to solve this condition, whereas the therapeutic strategies still in an expert-based opinion phase and there is no consensus yet. We report a case of SRPE in a 23-year-old man successfully treated using paroxetine.

\section{Case Report}

A 23-year-old Chinese college student reported suffering from recurrent painful nocturnal erection during sleep for 1 year. He woke up three to four times a night with a painful penile erection, the painful erection could be relieved by micturition. Sometimes, he felt genital soreness and burning after he woke up, lasting for 5-10 minutes. He has a normal painless penile erection during intercourse and masturbation. Frequently sleep loss made him anxious, attention impairment, irritable and extreme fatigue in the daytime. Then he sought medical help.
Previously, he had a consultation with the general practitioners, treated with tamsulosin $(0.2 \mathrm{mg}$ per night) for 4 weeks, without any effect. Then he was referred to our clinic to sought further management. Physical examination, blood and urine test, and brain MRI were performed, except the serum testosterone was $11.56 \mathrm{ng} / \mathrm{ml}$ (normal range 1.42-9.23); other results did not showed any abnormal. He denied taken any testosterone supplements or sexual stimulants. He refused further diagnostic examination such as polysomnography (PSG) and nocturnal penile tumescence and rigidity (NPT-R) monitoring due to the cost, however, his smart bracelet has a sleep monitoring function that can detect REM sleep and awake times. According to the smart bracelet, his sleep was fragmented by awakenings at the end of all the REM period (Figure 1), which is the main feature of sleep performance in patients with SRPE, combined with his painful erection every time he wakes up, SRPE was diagnosed. Priapism was excluded, for the reason it usually lasts for many hours and does not stop spontaneously, what's more, priapism is independent of sleep. In the beginning, he asked for non-pharmacological therapy. Taking into account his request and the side effects of the antiandrogens, which may induce hypogonadism state on sexual performance, the patient previously underwent highfrequency hyperthermia therapy half an hour a time every other day for two weeks, but there is no improvement for his condition. In the following month, he took zhibai dihuang wan (a traditional Chinese medicine composed of eight herbal ingredients), reexamination of 


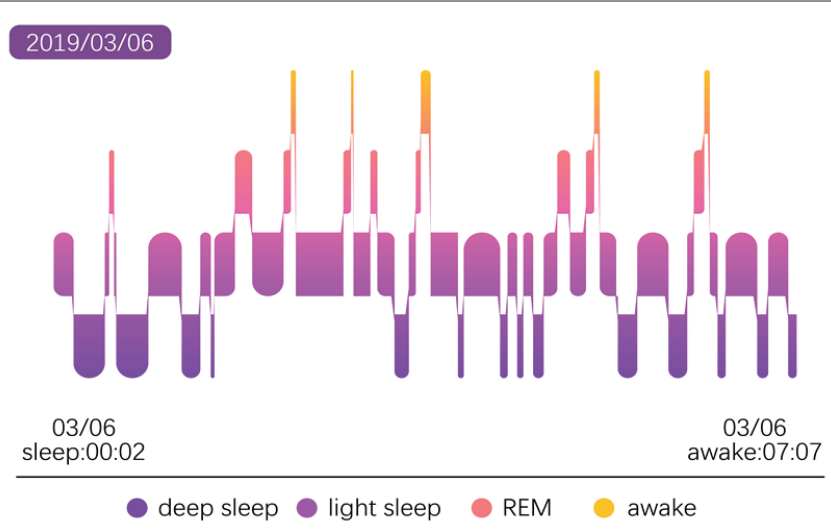

Figure 1: Before treatments (2019/03/06), severe fragmentation of REM sleep and 5 times of wakefulness were observed.

serum testosterone indicates that it had been reduced to the normal range $(6.4 \mathrm{ng} / \mathrm{ml})$, however, his symptoms persisted. What's more, unfortunately, his grandfather passed away two weeks later, he did not sleep for nearly 60 hours due to the customs and sadness mood, as a result, his condition became worse, the erection pain gradually increased to six to eight times a night, he felt extreme fatigue in the daytime. The psychological assessment scale suggests he was mild depression and mild anxiety, he scored 7 in patient health questionnaire 9 (normal value $<5$ ) and 8 in generalised anxiety disorder assessment (normal value $<5$ ). To get rid of this situation, he agrees to accept the treatment of antidepressants. Oral paroxetine $20 \mathrm{mg}$ taken at bed time. Two weeks later, both the frequency and intensity of the SRPE was decreased dramatically. After twelve weeks of the treatment, all symptoms were disappeared, no wakefulness was observed during the whole sleep (Figure 2), and he subsequently stopped the medication without recurrence of SRPE over the next four weeks. At-12-month follow-up, his only experienced noticeable nocturnal erections when he was very tired, but not accompanied by pain, he felt very satisfied.

\section{Discussion}

Nocturnal penile tumescence (NPT) is a physiological process that naturally occurs in all healthy males from early infancy into old age. It usually appears several times during rapid eye movement (REM) sleep, lasting for about 25 minutes. These erections have no association with sexual stimulation and dream content. Different from NPT, SRPE is characterized by recurrent painful nocturnal penile tumescence that occurs during REM sleep in the absence of pain during intercourse and wakefulness. SRPE was first described by Karacan I in 1971 [3], in the following years, several case reports and series of 47 cases had been described; this rare disease is gradually attracting more attention.

The pathophysiology of SRPE is still unknown, several hypotheses about this disorder have been proposed. Recently, the most adopted theory of pathophysiological mechanism behind SRPE is neurotransmitter regulation disorders during REM sleep. Cholinergic neurons produce acetylcholine in the REM phase, which activates the cavernous smooth muscle cholinergic $M$ receptor and releases nitric oxide, and then the cavernous smooth muscle endothelial cells relax and the penile arteries and cavernous sinus open, leading to blood engorgement and penile erection. While in SRPE patients, they may have hypersensitivity reaction during REM sleep and leads to excessive secretion of neurotransmitters, thus causing prolong penile tumescence [4]. This hypothesis may explain the increased erections, but still lacks the explanation of the associated pain. Engstrom M, et

\section{9/06/12}

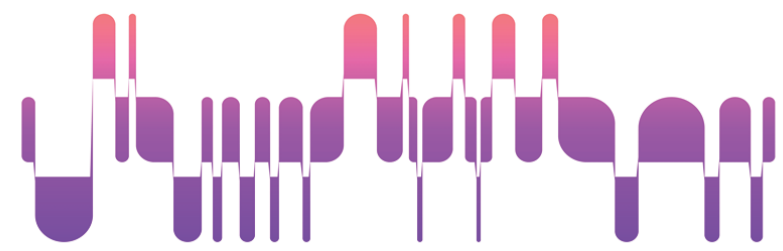

$06 / 12$

sleep:00:05

$06 / 12$

deep sleep light sleep REM awake

Figure 2: After treatments (2019/06/12), not any wakefulness were observed through the whole sleep.

al. [5] found that in patients in acute phase of sleep-related headache showed lower thresholds for heat, cold and pain than that of the normal control group, resulting in pain in REM sleep. SRPE almost also occurs in the REM phase, which is highly similar to patients with sleep-related headaches. It can be speculated that SRPE-related pain may be caused by hyperalgesia in the REM phase. Ferini-Strambi L, et al. [6] suggested that SRPE may be associated with the autonomic system, they observed a reduction in cardiac vagal activity and possible beta-adrenergic hyperactivity during sleep, which might explain the transient response of SRPE to beta-blockers. Serum testosterone levels were also considered associated with SPRE. Androgens regulate the contraction and relaxation of penile cavernous smooth muscle through mediate endothelial nitric oxide production. It had been observed in some hypogonadal men that the frequency and rigidity of NPT increased after testosterone supplement. However, no cases of SRPE were reported in the previous studies of patients with increased levels of serum testosterone [7]. The treatment with antiandrogens usually ineffective [8]. In our case, the serum testosterone level was higher than normal, after medical treatment, his serum testosterone level became normal while painful nocturnal erections were still existed. In addition, Obstructive sleep apnea syndrome [9] and neurovascular compression of basal forebrain [10] are also potential causes of SRPE that cannot be ignored.

The diagnosis of SRPE mainly depends on clinical features, since it is such a rare disease, the differential diagnosis is very necessary. Other medical conditions that can cause painful erections such as phimosis, peyronie's disease and priapism must be excluded. PSG combined with NPT-R is helpful to make the diagnosis. Patients with SRPE performed PSG and NPT-R may show some typical characteristics: fragmented of REM sleep and all REM episodes accompanied by erection and subsequent awakening. The smart bracelet that can monitor sleep can also play a similar role to PSG.

Many drugs have been used to prevent this disorder; however, there is no one effective definitive treatment for it. The potential pharmacological treatments include antidepressants (paroxetine), muscle relaxants (baclofen), Phosphodiesterase type 5 (PED5) inhibitors and antipsychotics (clozapine).

Antidepressants inhibit the downregulation or synaptic reuptake of serotonin, result in an anticholinergic and sedative effect. These drugs showed variable results in patients with SRPE [11]. 1 patient treated with clomipramine had a permanent beneficial effect, 4 patients (out of 8) had a short-term effect while treated with amitriptyline, 
and almost all the patients have taken phenelzine, paroxetine and clomipramine showed a transient efficacy. Imipramine, trimipramine were not effective in any cases.

In the previous literature, there were only 2 papers $[6,12]$ on the treatment of SRPE with paroxetine, and they were all published more than 20 years ago. In a recent review of SRPE, the antidepressants to which paroxetine belongs were even included as one of the abandoned treatments by the authors. In view of this, paroxetine seems to have lost its value for SRPE. However, in our reported case, we successfully treated SRPE with paroxetine and achieved a long-term and stable effect. The underlying mechanism may be paroxetine increases levels of serotonin in the brain by preventing the reuptake of serotonin by nerve cells, as a result, reducing muscle tone and suppressing erection during REM sleep.

Baclofen is a skeletal muscle relaxant that is traditionally used for spasticity, it had been tried on 17 patients with SRPE in 3 case series and 1 case report [11], except 1 patient did not respond, and other 16 patients had been relieved to vary degrees. 6 of 17 patients were reported a complete remission of symptoms. The theoretical benefit of baclofen could be based on the suppression of glutamate release and relaxation of the ischiocavernosous and bulbospongiosus muscles.

PED5 inhibitors increases the cyclic guanosine monophosphate (cGMP) level and consequently promote erection, they have been wildly used in patients with erectile dysfunction. Paradoxically, according to some studies, daily low doses of PDE5 inhibitors in the long term may increase basal level of cGMP, causing restoration of PDE5 expression and activity, resulting in a decreased erection. 2 patients with SRPE had been successfully treated by low dose $(5 \mathrm{mg}$ daily) of tadalafil and reported no side effects [8].

In the literature, clozapine was used in 2 cases of SRPE $[10,13]$, with sustainable response in one case. The patient was successfully treated with low dose of clozapine. This drug is a neuroleptic with anticholinergic and sedative effects, the dangerous side effects such as myocarditis and agranulocytosis make it less encouraging to apply in SRPE patients.

\section{Conclusions}

SRPE is such a poorly recognized disease that most patients have a mean patient-doctor delay of 5 years between symptoms onset and diagnosis [2]. Neurotransmitter regulation disorders during REM sleep were supposed to be the potential pathophysiological mechanism of SRPE, in addition, our case suggested that there was no significant correlation between serum testosterone levels and SRPE. PSG combined with NPT-R can make the diagnose of SRPE, in this case, the patient refused PSG and NPT-R test, we make the diagnosis based on sleep status as measured by the smart bracelet, that is a limitation of our report, even though the smart bracelet which is cheap and easy to operate can be used as a substitute for PSG to some extent when SRPE is suspected, NPT and PSG examination should be carried out as far as possible, for the more comprehensive information they can provide. Baclofen is the most effective drug in the previous reports. In our case, paroxetine also showed a long-term and stable effect on patients with SRPE, it indicates that the therapeutic effect of paroxetine on SRPE deserves further study and observation.

\section{References}

1. American Academy of Sleep Medicine (2014) International classification of sleep disorders, $3^{\text {rd }}$ ed, Darien IL: American Academy of Sleep Medicine.

2. Abdessater M, Kanbar A, Zugail AS, Hammadi AA, Guillonneau B, et al. (2019) Sleep related painful erection: an algorithm for evaluation and management. Basic Clin Androl 29: 15.

3. Karakan I (1971) Painful nocturnal penile erections. JAMA 215: 1831-1834.

4. Schmidt MH (2018) Sleep-related erection neurophysiology: a journey of discovery. Sleep Med 49: 24-27.

5. Engstrøm M, Hagen K, Bjørk MH, Stovner LJ, Sand T (2014) Sleep quality and arousal in migraine and tension-type headache: the headache-sleep study. Acta Neurol Scand Suppl 198: 47-54.

6. Ferini-Strambi L, Oldani A, Zucconi M, Castronovo V, Montorsi F, et al. (1996) Sleep-related painful erections: clinical and polysomnographic features. J Sleep Res 5: 195-197.

7. Granata AR, Rochira V, Lerchl A, Marrama P, Carani C (1997) Relationship between sleep-related erections and testosterone levels in men. J Androl 18: 522-527.

8. Vreugdenhil S, Weidenaar AC, de Jong IJ, van Driel MF (2017) SleepRelated Painful Erections-A Case Series of 24 Patients Regarding Diagnostics and Treatment Options. Sex Med 5: e237-e243.

9. Abouda M, Jomni T, Yangui F, Charfi MR, Arnulf I (2016) SleepRelated Painful Erections in a Patient with Obstructive Sleep Apnea Syndrome. Arch Sex Behav 45: 241-245.

10. Szücs A, Janszky J, Barsi P, Erdei E, Clemens Z, et al. (2002) Sleep-related painful erection is associated with neurovascular compression of basal forebrain. J Neurol 249: 486-487.

11. Vreugdenhil S, Weidenaar AC, de Jong IJ, van Driel MF (2018) SleepRelated Painful Erections: A Meta-Analysis on the Pathophysiology and Risks and Benefits of Medical Treatments. J Sex Med 15: 5-19.

12. López VM, Rufete AM, Chaparro LP, Llopis JAG, Puentes CF, et al. (1999) Painful erections related to sleeping. Actas Urol Esp 23: 53941.

13. Steiger A, Benkert O (1989) Examination and treatment of sleeprelated painful erections--a case report. Arch Sex Behav 18: 263267. 